FERMILAB-Pub-93/378-A

January, 1994

(revised version)

\title{
Mass and radius of cosmic balloons
}

\author{
Yun Wang \\ NASA/Fermilab Astrophysics Center \\ Fermi National Accelerator Laboratory, Batavia, IL 60510-0500
}

\begin{abstract}
Cosmic balloons are spherical domain walls with relativistic particles trapped inside. We derive the exact mass and radius relations for a static cosmic balloon using Gauss-Codazzi equations. The cosmic balloon mass as a function of its radius, $M(R)$, is found to have a functional form similar to that of fermion soliton stars, with a fixed point at $2 G M(R) / R \simeq 0.486$, which corresponds to the limit of infinite central density. We derive a simple analytical approximation for the mass density of a spherically symmetric relativistic gas star. When applied to the computation of the mass and radius of a cosmic balloon, the analytical approximation yields fairly good agreement with the exact numerical solutions.
\end{abstract}




\section{Introduction}

A particle with a discrete symmetry may have a different mass on either side of the domain wall, which may form in an early Universe phase transition with the spontaneous breaking of the discrete symmetry. When a particle has less energy than the rest mass it would have on the other side of the wall, it can become trapped inside a region with a closed domain wall as the boundary. Closed domain walls filled with such particles have been named "cosmic balloons" [1]. Due to effects such as the emission of gravitational radiation, irregular shapes of balloons oscillate and eventually settle down to spheres. Here we are only concerned with a spherical cosmic balloon.

Cosmic balloons are stable objects. The pressure of the relativistic gas inside the balloon balances the surface tension of the wall. An incarnation of a cosmic balloon is a neutrino ball [2], which is a spherical domain wall with light right-handed neutrinos trapped inside. Details of neutrino balls have been worked out which seem to indicate that they are plausible astrophysical objects [2], possibly providing an alternative explaination for the mass of quasars and other astrophysical phenomena.

In this work, we follow Ref[ 1 in the study of general properties of cosmic balloons, in particular, the mass of the balloon as a function of its radius. The analytical approximation in Ref[1] has an extremely involved numerical form, and the mass versus radius curve found there is numerically incomplete. In another previous work with similar objectives, Ref[3], an analytical approximation was applied beyond its valid range and led to incorrect numerical solutions of the mass and radius. In this work, we find simple and transparent analytical expressions which are useful in helping us understand the cosmic balloon solutions qualitatively, and we compute the complete set of mass and radius numerically with and without the application of the analytical approximation. 


\section{Mass and radius relations}

To find the radius $R$ and mass $M$ of a cosmic balloon, we study the static configuration of a spherical domain wall containing gas. Let the metric inside the domain wall $(r<R)$ be

$$
d \tau^{2}=B(r) d t^{2}-A(r) d r^{2}-r^{2}\left(d \theta^{2}+\sin ^{2} \theta d \phi^{2}\right) .
$$

The motion of spherical domain walls containing vacuum has been solved in Ref.(何), using the Gauss-Codazzi formalism. Following Ref.(近), we find the equations of motion for a spherical domain wall containing a perfect fluid with pressure $p$ and density $\rho$ :

$$
\begin{aligned}
(\alpha+\beta) \ddot{R}+\frac{\alpha G M}{R^{2}}+\frac{\beta}{2}\left[\frac{A^{\prime}}{A} \dot{R}^{2}+\frac{B^{\prime}}{B} \alpha^{2}\right] & =-\alpha \beta\left[\frac{2(\alpha+\beta)}{R}-\frac{2 p}{\sigma}-\frac{2 \dot{R}^{2}}{\sigma} A(\rho+p)\right] \\
(\alpha-\beta) \ddot{R}+\frac{\alpha G M}{R^{2}}-\frac{\beta}{2}\left[\frac{A^{\prime}}{A} \dot{R}^{2}+\frac{B^{\prime}}{B} \alpha^{2}\right] & =-4 \alpha \beta \pi G \sigma,
\end{aligned}
$$

Here a dot denotes a derivative with respect to proper time, and a prime denotes a derivative with respect to $r=R$. We have defined

$$
\begin{aligned}
& \alpha \equiv\left[A^{-1}(R)+\dot{R}^{2}\right]^{1 / 2}, \\
& \beta \equiv\left[1-\frac{2 G M}{R}+\dot{R}^{2}\right]^{1 / 2} .
\end{aligned}
$$

$M$ is the total mass of a cosmic balloon of radius $R$.

The equation for hydrostatic equilibrium is [5]

$$
\frac{B^{\prime}}{B}=-\frac{2 p^{\prime}}{p+\rho} \text {. }
$$

This and the Einstein equations can be combined to give a single differential equation:

$$
-r^{2} p^{\prime}(r)=G \mathcal{M}(r) \rho(r)\left[1+\frac{p(r)}{\rho(r)}\right]\left[1+\frac{4 \pi r^{3} p(r)}{\mathcal{M}(r)}\right]\left[1-\frac{2 G \mathcal{M}(r)}{r}\right]^{-1},
$$

where we have defined $\mathcal{M}(r)$ such that

$$
\mathcal{M}^{\prime}(r)=4 \pi r^{2} \rho(r), \quad \mathcal{M}(0)=0 .
$$


For $A(0)$ finite,

$$
A(r)=\left[1-\frac{2 G \mathcal{M}(r)}{r}\right]^{-1} .
$$

Using the above well known results in Eqs.(2.2) and setting $\dot{R}=0=\ddot{R}$, we find

$$
\begin{aligned}
& M(R)=\mathcal{M}(R)+2 \pi R^{2} \sigma(\alpha+\beta), \\
& p(R)=\frac{\sigma}{2 R}\left(3 \alpha+\frac{1}{\beta}\right)
\end{aligned}
$$

with

$$
\alpha=\left[1-\frac{2 G \mathcal{M}(R)}{R}\right]^{1 / 2}, \quad \beta=\left[1-\frac{2 G M}{R}\right]^{1 / 2} .
$$

Eq.(2.8) can be rewritten to give

$$
\beta=\alpha-4 \pi R G \sigma, \quad \text { for } R<\frac{\alpha}{4 \pi G \sigma} .
$$

A cosmic balloon with $R>\alpha /(4 \pi G \sigma)$ is contained within its Schwarzschild radius, a case in which we are not interested here. Hence

$$
M(R)=\mathcal{M}(R)+4 \pi R^{2} \sigma[\alpha-2 \pi R G \sigma]
$$

Note that $\mathcal{M}(R)$ is the total energy of the matter and the gravitational field inside the cosmic balloon [5]. Eq. 2.9) gives the radius $R$ of a cosmic balloon as a function of its surface tension $\sigma$, it can be rewritten as

$$
p(R)=\frac{2 \alpha \sigma}{R}+\frac{G \sigma \mathcal{M}(R)}{\beta R^{2}}+\frac{\alpha}{\beta} 2 \pi G \sigma^{2} .
$$

We see that the gas pressure at $R$ is balanced by the surface tension of the domain wall, the gravitational force on the wall due to the total mass inside the wall and the wall itself. 


\section{An exact solution}

Let us define dimensionless variables as follows:

$$
\begin{aligned}
x(r) & \equiv \frac{2 G \mathcal{M}(r)}{r}, & & \\
\bar{r} & \equiv \frac{r}{r_{0}}, & & \left(r_{0} \equiv[48 \pi G \sigma]^{-1}\right), \\
\rho^{*}(r) & \equiv \frac{\rho(r)}{\rho_{0}}, & & \left(\rho_{0} \equiv\left[8 \pi G r_{0}^{2}\right]^{-1}\right), \\
\bar{M}(R) & \equiv \frac{M(R)}{M_{0}}, & & \left(M_{0} \equiv \frac{r_{0}}{2 G}\right) .
\end{aligned}
$$

Note that the length scale $r_{0}$ and the mass scale $M_{0}$ can be written as

$$
\begin{aligned}
& r_{0} \simeq 1.95 \times 10^{13} \mathrm{~cm}\left[\frac{\sigma}{(\mathrm{TeV})^{3}}\right]^{-1}, \\
& M_{0} \simeq 6.6 \times 10^{7} M_{\odot}\left[\frac{\sigma}{(\mathrm{TeV})^{3}}\right]^{-1} .
\end{aligned}
$$

This is interesting since both $\bar{R}$ and $\bar{M}(R)$ have maxima on the order of 1 , as we shall see. $\sigma^{1 / 3}$ is usually associated with the scale of the discrete symmetry breaking, but it could be made anything depending on the particular model of cosmic balloon.

Eq.(2.5) can be rewritten as

$$
x^{\prime \prime}=-\frac{2}{3} \frac{1}{1-x}\left[x^{\prime 2}+5 x^{\prime} f_{1}(\bar{r})+(7 x-3) f_{2}(\bar{r})\right],
$$

where the primes denote differentiation with respect to $\bar{r}$, and

$$
\begin{array}{ll}
f_{1}(\bar{r}) \equiv \frac{x}{\bar{r}}, & f_{1}(0)=0, \\
f_{2}(\bar{r}) \equiv \frac{x}{\bar{r}^{2}}, & f_{2}(0) \equiv q=\frac{8 \pi G \rho(0) r_{0}^{2}}{3}, \\
x(0)=0=x^{\prime}(\bar{r}=0), & \text { if } \rho(0) \text { is finite. }
\end{array}
$$

Note that $\rho(0)$ dependence only comes in through the boundary condition of the function $f_{2}(\bar{r})$. In terms of $x$ and $\bar{r}$,

$$
\rho^{*}(r)=\frac{1}{\bar{r}^{2}}\left[x+\bar{r} x^{\prime}\right] .
$$


The scaled radius of the cosmic balloon $\bar{R}$ is given by the intersection of $\rho^{*}(r)$ and

$$
\rho^{*}(R)=\frac{1}{4 \bar{R}}\left(3 \alpha+\frac{1}{\beta}\right) .
$$

The scaled mass of the cosmic balloon is

$$
\bar{M}(R)=\bar{R}\left[x(\bar{R})+\frac{\bar{R}}{6}\left(\alpha-\frac{\bar{R}}{24}\right)\right] .
$$

Here

$$
\alpha=[1-x(R)]^{1 / 2}, \quad \beta=\alpha-\bar{R} / 12, \quad \text { for } \bar{R}<12 \alpha .
$$

Eq.(3.3) has an obvious solution [5]

$$
x=\frac{3}{7}, \quad \rho(r)=\frac{3}{56 \pi G r^{2}} \text { or } \rho^{*}(r)=\frac{3}{7 \bar{r}^{2}},
$$

which has infinite central mass density. The corresponding cosmic balloon radius and mass are:

$$
\begin{aligned}
& \bar{R}=\frac{4}{\sqrt{7}}(5-\sqrt{22}) \simeq 0.468 \\
& \bar{M}(R)=\frac{8(122-25 \sqrt{22})}{63 \sqrt{7}} \simeq 0.2275, \\
& \frac{2 G M(R)}{R}=\frac{\bar{M}(R)}{\bar{R}}=\frac{2(20-\sqrt{22})}{63} \simeq 0.486 .
\end{aligned}
$$

\section{Numerical solutions}

The numerical solutions of the cosmic balloon mass function can be obtained in a straightforward way. It is most convenient to use the parameter $q$ to track the solutions of Eq.(3.3). Note that

$$
q \equiv \frac{8 \pi G \rho(0) r_{0}^{2}}{3} \propto \rho(0), \quad \rho^{*}(0)=3 q .
$$

For a given value of $q$, Eq. (3.3) can be integrated to find $x(r)$ and hence $\rho^{*}(r) \cdot \rho^{*}(r)$ and $\rho^{*}(R)=\left(3 \alpha+\beta^{-1}\right) / 4 \bar{R}$ have two intersections for $q>q_{c}$, which give two values of the 
cosmic balloon radius $R$, corresponding to two different branches of the cosmic balloon mass/radius solutions (see Fig. 1). The two branches of solutions meet at $q=q_{c} \simeq 1$, for $q<q_{c}$ (or $\left.\rho(0)<\rho_{c}(0)\right)$ there are no solutions and cosmic balloons do not exist. $q_{c}$ corresponds to the minimum of the central mass density for a cosmic balloon.

Fig.2 and Fig.3 are the radius $R$ and mass $M(R)$ versus $q$ respectively. For increasing $q$ (i.e., $\rho(0)$ ), the first branch of solutions gives decreasing $R$ and $M(R)$; the second branch of solutions gives $R$ and $M(R)$ with complex and similar behavior. In the second branch of solutions, $R$ increases until it reaches the maximum $\left(\bar{R}_{m} \simeq 0.732\right)$, then it decreases until it reaches the minimum, it then increases again until it reaches a second (smaller) maximum, then it decreases again to a second (larger) minimum, and continues in this manner. From Fig. 2, it is clear that $R$ oscillates with damping amplitude around $\bar{R} \simeq 0.468$ for large $q . M(R)$ has the same behavior but with a phase lag in $q[\rho(0)]$ compared to $R\left(\bar{M}_{m} \simeq 0.374, \bar{R}_{M m} \simeq 0.71\right)$, and it oscillates around $\bar{M}(R) \simeq 0.2275$ for large $q$ (see Fig. 3). This is not surprising, since $\bar{R} \simeq 0.468$ and $\bar{M}(R) \simeq 0.2275$ correspond to the exact solution of $\rho(r)$ in the limit of $\rho(0) \rightarrow \infty$ (i.e., $q \rightarrow \infty$ ). In the plot of $M(R)$ versus $R$ (Fig. 4), we see that the mass function spirals in toward the fixed point $(\bar{R} \simeq 0.468, \bar{M}(R) \simeq 0.2275)$, which satisfies $2 G M(R) / R \simeq 0.486$.

The maximum mass and the corresponding radius for a cosmic balloon are

$$
\begin{aligned}
& M_{\text {max }}=\bar{M}_{m} M_{0} \simeq 2.47 \times 10^{7} M_{\odot}\left[\frac{\sigma}{(\mathrm{TeV})^{3}}\right]^{-1}, \\
& R_{M \max }=\bar{R}_{M m} r_{0} \simeq 1.38 \times 10^{13} \mathrm{~cm}\left[\frac{\sigma}{(\mathrm{TeV})^{3}}\right]^{-1}
\end{aligned}
$$

Since $2 G M(R) / R=\bar{M}(\bar{R}) / \bar{R}$, the fractional red shift $z$ of a spectral line emitted from the surface of a cosmic balloon [5]

$$
z \equiv \frac{\Delta \lambda}{\lambda}=\left(1-\frac{2 G M}{R}\right)^{-1 / 2}-1
$$


is independent of the actual physical scales $r_{0}$ and $M_{0}$, i.e., independent of $\sigma$ (the surface tension of the domain wall). The exact numerical solution shows that $2 G M / R$ reaches maximum $\left((2 G M / R)_{\max } \simeq 0.56\right)$ after the mass has already reached maximum. Before $M=M_{\max }, 2 G M / R$ increases with increasing $M$. The maximum of mass then corresponds to the maximum of the fractional red shift $z$ for a stable cosmic balloon, $z_{\max } \simeq 0.46\left(2 G M_{m} / R_{M m} \simeq 0.53\right)$. At the minimum of the central mass density $\left(\bar{R}_{c} \simeq 0.56, \bar{M}_{c} \simeq 0.17,2 G M_{c} / R_{c} \simeq 0.30\right), z_{c} \simeq 0.20$.

\section{Analytical approximations}

To obtain useful analytical approximations, we scale Eq.(3.3) such that its solutions are independent of the central mass density $\rho(0)$ (i.e., independent of $q$ ). Define

$$
t=\sqrt{3 q} \bar{r}
$$

Eq.(3.3) becomes

$$
\ddot{x}=-\frac{2}{3} \frac{1}{1-x}\left[\dot{x}^{2}+5 \dot{x} f_{1}^{*}(t)+(7 x-3) f_{2}^{*}(t)\right],
$$

where the dots denote differentiation with respect to $t$, and

$$
\begin{array}{ll}
f_{1}^{*}(t) \equiv \frac{x}{t}, & f_{1}^{*}(0)=0, \\
f_{2}^{*}(t) \equiv \frac{x}{t^{2}}, & f_{2}^{*}(0) \equiv \frac{1}{3}, \\
x(t=0)=0=\dot{x}(t=0) .
\end{array}
$$

Note that Eq.(5.1) is exactly the same in form as Eq.(3.3), but without $\rho(0)$ dependence in the boundary conditions. Correspondingly,

$$
\bar{\rho}(t) \equiv \frac{\rho^{*}(r)}{\rho^{*}(0)}=\frac{\rho(r)}{\rho(0)}=\frac{1}{t^{2}}[x+t \dot{x}]
$$


Combining Eqs.(5.3) and (5.1), we can express $x(t)$ in terms of $\bar{\rho}(t)$ :

$$
x=\frac{[(\dot{\bar{\rho}} / \bar{\rho})+2 \bar{\rho} t / 3] t}{(\dot{\bar{\rho}} / \bar{\rho}) t-2} .
$$

The radius of the cosmic balloon is now given by

$$
\bar{\rho}\left(t_{R}\right)=\frac{1}{4 \sqrt{3 q} t_{R}}\left(3 \alpha\left(t_{R}\right)+\frac{1}{\beta\left(t_{R}\right)}\right),
$$

where $t_{R} \equiv \sqrt{3 q} \bar{R}$, and

$$
\begin{aligned}
& \alpha\left(t_{R}\right)=\left[1-x\left(t_{R}\right)\right]^{1 / 2}, \\
& \beta\left(t_{R}\right)=\alpha\left(t_{R}\right)-\frac{t_{R}}{12 \sqrt{3 q}} .
\end{aligned}
$$

Given the function $\bar{\rho}(t)$, we can find $R(q)$. The mass of a cosmic balloon is (see Eq.(3.7))

$$
\bar{M}(R)=\bar{R}\left[x\left(t_{R}\right)+\frac{\bar{R}}{6}\left(\alpha\left(t_{R}\right)-\frac{\bar{R}}{24}\right)\right] .
$$

Recall that $\bar{M}(R)$ and $\bar{R}$ are defined in Eq.(3.1).

The analytical solution from Ref[3]

$$
\bar{\rho}_{1}(t)=\frac{1}{\cosh ^{2}(k t)}, \quad k=\sqrt{\frac{2}{3}}
$$

is an extremely good approximation for $t \lesssim 1$. The corresponding $x(t)$ is

$$
x_{1}(t)=\frac{(k t) \tanh (k t)-t^{2} /\left[3 \cosh ^{2}(k t)\right]}{(k t) \tanh (k t)+1} .
$$

Eq.(5.1) still has the exact solution $x=3 / 7$, which has the wrong boundary conditions at $t=0$. However, the solution with the correct boundary conditions at $t=0$ should approach $x=3 / 7$ in the limit of large $t$, as indicated by our exact numerical solutions. To the second order of a small parameter $A$, the perturbation around $x=3 / 7$ gives

$$
\begin{aligned}
x_{2}(t)= & \frac{3}{7}+\frac{A}{t^{3 / 4}} \cos [\theta(t)]+ \\
& \frac{7}{32} \frac{A^{2}}{t^{3 / 2}}\left\{-7+\frac{139}{54} \cos [2 \theta(t)]-\frac{19}{54} \sqrt{47} \sin [2 \theta(t)]\right\},
\end{aligned}
$$


where

$$
\theta(t)=\frac{\sqrt{47}}{4} \ln \left(\frac{t}{3 / 7}\right)+\tan ^{-1}\left(\frac{1}{\sqrt{47}}\right)-\frac{\pi}{B} .
$$

$A$ and $B$ are constants which are independent of $\rho(0)$. A good fit of Eq.(5.10) with the exact numerical solution gives

$$
A \simeq-0.18, \quad B \simeq 3.54
$$

Eq.(5.3) gives the corresponding $\bar{\rho}(t)$ for $x_{2}(t)$ :

$$
\begin{aligned}
\bar{\rho}_{2}(t)= & \frac{1}{t^{2}}\left[\frac{3}{7}+\frac{A}{4 t^{3 / 4}}(\cos [\theta(t)]-\sqrt{47} \sin [\theta(t)])\right. \\
& \left.+\frac{7}{32} \frac{A^{2}}{t^{3 / 2}}\left(\frac{7}{2}-\frac{86}{9} \cos [2 \theta(t)]-\frac{10}{9} \sqrt{47} \sin [2 \theta(t)]\right)\right] .
\end{aligned}
$$

Since Eq.(5.8) is valid for small $t$, while Eq.(5.13) is valid for large $t$, we can construct an approximate solution by combining the two solutions with appropriate weight functions, i.e.

$$
\bar{\rho}(t)=w_{1}(t) \bar{\rho}_{1}(t)+w_{2}(t) \bar{\rho}_{2}(t)
$$

where $w_{1}(t)$ and $w_{2}(t)$ are weight functions. For example

$$
w_{1}(t)=\frac{1}{1+e^{t / n_{1}} t^{n_{2}}}, \quad W_{2}(t)=\frac{t^{n_{2}}}{e^{-t / n_{1}}+t^{n_{2}}} .
$$

A good choice is

$$
n_{1}=20, \quad n_{2}=8 .
$$

The cosmic balloon radius as a function of the central mass density $\rho(0)$ can be computed by using Eqs.(5.14) and (5.5). We can write

$$
x(t)=w_{1}(t) x_{1}(t)+w_{2}(t) x_{2}(t) .
$$

The mass of the cosmic balloon is given by Eq.(5.7). 
In Fig.1, we see that Eq.(5.14) is an extremely good approximation of the exact mass density $\bar{\rho}(t)$ (scaled to be independent of $\rho(0)$ ). The radius $R$ and mass $M(R)$ obtained by using Eqs.(5.14) and (5.17) are plotted in dashed lines in Figs. 2-4, they are quite close to the exact solutions.

\section{Remarks}

The spiral behaviour in $M(R)$ versus $R$ of cosmic balloons (see Fig.4) resembles that of fermion stars [6]. This is not surprising since cosmic balloons and soliton stars are similar objects. $d M / d \rho(0)=0$ generally signifies the change from stability to instability (or vice versa), and $d M / d \rho(0)>0$ indicates stability. However, due to dynamical complications, the range of $M(R)$ and $R$ beyond the first mass maximum $(d M / d \rho(0)=0)$ on the curve in Fig. 4 does not correspond to stable configurations of cosmic balloons, although the conditions for stability are satisfied each time the curve bends upward (for increasing $\rho(0))$. It is possible that the existence of the fixed point $(\bar{R} \simeq 0.468, \bar{M}(R) \simeq 0.2275$, $2 G M(R) / R \simeq 0.486$ ) indicates that the cosmic balloons beyond the stability range oscillate around a finite configuration with $2 G M(R) / R \simeq 0.486$, instead of collapsing into black holes.

\section{Acknowledgments}

I thank R. Caldwell and V. Silveira helpful discussions. This work was supported by the DOE and NASA under Grant NAGW-2381. 


\section{References}

1. B. Holdom, Univ of Toronto preprint \#UTPT-93-18 (1993).

2. B. Holdom, Phys. Rev. D 36 (1987) 1000; A.D. Dolgov and O. Yu. Markin, Sov. Phys. JETP 71 (1990) 207; A.D. Dolgov and O. Yu. Markin, Prog. Theor. Phys. 85 (1991) 1091; B. Holdom and R.A. Malaney, CITA preprint CITA/93/22 (1993).

3. R. Mańka, I. Bednarek, and D. Karczewska, Univ of Silesia preprint UŚL-TH-93-01.

4. J. Ipser and P. Sikivie, Phys. Rev. D 30 (1984) 712.

5. S. Weinberg, Gravitation and Cosmology, John Wiley and Sons, 1972.

6. T.D. Lee and Y. Pang, Phys. Rev. D 35 (1987) (3678); T.D. Lee, Phys. Rep. 221 (1992) 251. 


\section{Figure Captions}

Fig. 1. $\bar{\rho}(t)$ and $\bar{\rho}\left(t_{R}\right)=\left[3 \alpha\left(t_{R}\right)+\beta^{-1}\left(t_{R}\right)\right] /\left(4 \sqrt{3 q} t_{R}\right)$, with $t \equiv \sqrt{3 q} \bar{r}$ and $q \equiv$ $8 \pi G \rho(0) r_{0}^{2} / 3=0.9,2,10$ (dotted lines from right to left). The dashed line is the analytical approximation of $\bar{\rho}(t)$ from Eq.(5.14), it almost overlaps with the exact solution (the solid line).

Fig. 2. $\bar{R}$ versus $q \equiv 8 \pi G \rho(0) r_{0}^{2} / 3$. The solid line is the exact solution, while the dashed line is the result of analytical approximation. The dotted line is $\bar{R}=0.468$.

Fig. 3. $\bar{M}(\bar{R})$ versus $q \equiv 8 \pi G \rho(0) r_{0}^{2} / 3$. The solid line is the exact solution, while the dashed line is the result of analytical approximation. The dotted line is $\bar{M}(R)=0.2275$.

Fig. 4. $M(R)$ versus $R$. The solid line is the exact solution, while the dashed line is the result of analytical approximation. 
This figure "fig1-1.png" is available in "png" format from: http://arxiv.org/ps/astro-ph/9312021v2 
This figure "fig2-1.png" is available in "png" format from: http://arxiv.org/ps/astro-ph/9312021v2 
This figure "fig1-2.png" is available in "png" format from: http://arxiv.org/ps/astro-ph/9312021v2 
This figure "fig2-2.png" is available in "png" format from: http://arxiv.org/ps/astro-ph/9312021v2 